\title{
Détentions et déportation à la frontière entre le Mexique et les Etats-Unis (partie 1)
}

\section{Samuel Schmidt}

\section{OpenEdition \\ Journals}

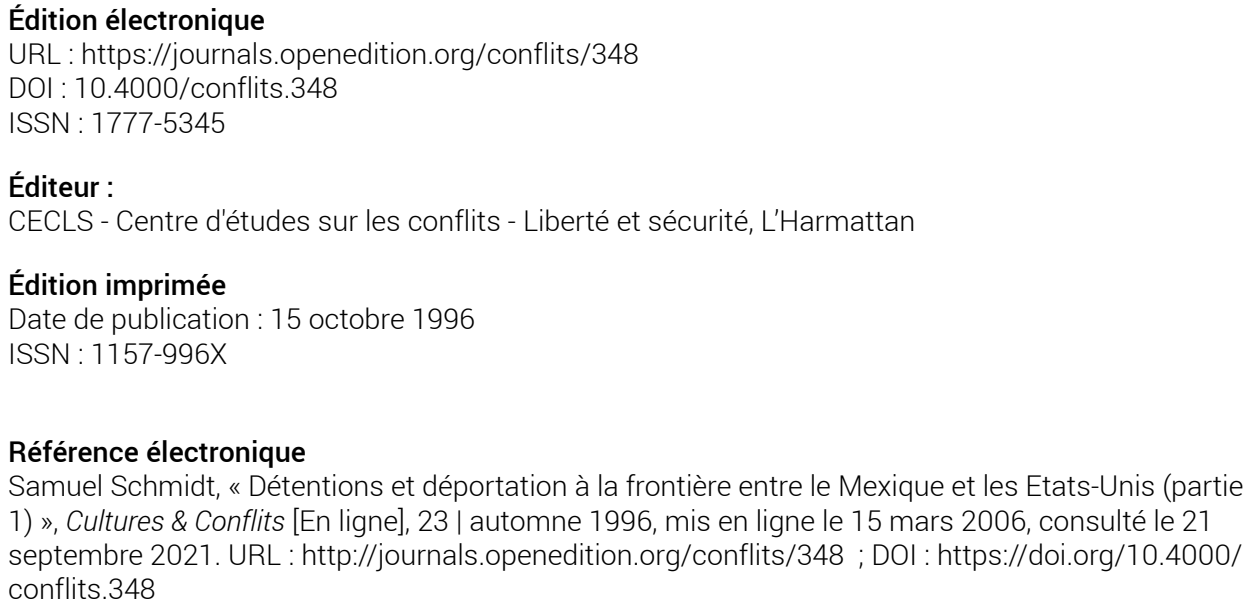

Ce document a été généré automatiquement le 21 septembre 2021.

Creative Commons License 


\title{
Détentions et déportation à la frontière entre le Mexique et les Etats-Unis (partie 1)
}

\author{
Samuel Schmidt
}

Samuel SCHMIDT ${ }^{1}$

Introduction

Les chiffres relatifs à l'émigration sont froids et instructifs. Mais il nous reste encore beaucoup à apprendre sur le drame humain que vivent ceux qui décident d'émigrer, en s'exilant et laissant derrière eux, leur famille, leur société, leur culture et jusqu'à leur langue.

Les migrations ne sont pas un phénomène nouveau, l'être humain était, au début, un nomade. La sédentarisation a favorisé l'idée que l'individu doit progresser et se développer sans effectuer de grands déplacements, par conséquent dès qu'une migration importante a lieu il convient de rechercher les causes qui expliquent un tel mouvement.

Il paraît compréhensible que les guerres ou les crises politiques fassent fuir les personnes, mais il semble plus difficile de comprendre la raison pour laquelle des millions d'individus quittent leur pays surtout lorsque ce dernier connaît depuis des décennies une certaine stabilité politique. On peut donc supposer que le Mexicain (comme l'émigrant de l'Amérique Centrale) doit se trouver dans une situation désespérée, pour oser s'aventurer à émigrer vers le nord, en courant le risque d'être traquer, persécuter, et celui de subir l'abus d'autorité de la police des deux pays².

Les réactions gouvernementales face à l'immigration illégale sont pour la plupart de caractère policier, bien que l'immigration ne soit pas une activité criminelle et que la frontière entre le Mexique et les Etats-Unis ne constitue pas une exception. Les mesures policières n'ont été efficaces d'aucun côté. Elles ont au contraire créé des tensions internes et externes, ainsi qu'un détournement du rôle que devraient jouer les contrôles frontaliers. Ce constat ne paraît guère décourager les hommes politiques qui 
présentent l'immigration comme un problème d'importance national contre lequel il faut se battre.

La criminalisation de l'immigration ${ }^{3}$ provoque une militarisation des frontières et constitue ainsi une barrière à l'intégration nationale en exacerbant des animosités et des sentiments raciaux dirigés contre les minorités ethniques. Cette criminalisation a encouragé par effet pervers la naissance d'activités criminelles, telle la contrebande, ou a favorisé l'émergence de bandes miliciennes prêtes à traquer les migrants sans papiers d'identité qui s'aventurent à travers les frontières ou les zones non protégées pendant la nuit.

La frontière entre le Mexique et les Etats-Unis a vu surgir une violence frontalière, source d'irritation des deux côtés de la frontière et a créé une situation d'insécurité sur la frange frontalière car les bandes qui attaquent les migrants étendent également leurs activités criminelles aux résidents, et d'autres bandes miliciennes utilisent cette frange territoriale pour commettre leur délits ou leurs rites d'initiation ${ }^{4}$.

Les récents flux migratoires de grande ampleur sont la manifestation la plus nette des importantes inégalités économiques. Le grand courant migratoire du Mexique vers les Etats-Unis manifeste le degré de misère qui règne au Mexique et le niveau de désespoir qu'ont atteint des millions de personnes qui, peut-être, ne pensent pas pouvoir résoudre leurs problèmes économiques dans leurs lieux d'origine.

L'économie mexicaine a été dépendante de celle des Etats-Unis pendant des décennies, de sorte que les deux pays portent la responsabilité du niveau et de la nature de la misère mexicaine. Cette relation n'est pas considérée aux Etats-Unis comme un élément valable pour normaliser la politique migratoire, qui semble même détachée de la « géo-économie ». Le maniement conjoncturel des décisions migratoires en est peutêtre la raison: on ouvre la frontière s'il y a carence de main d'oeuvre et on ferme dès que le marché se sature, bien qu'incidemment cette pratique soit justifiée par des critères politico-idéologiques ${ }^{5}$.

Il est important actuellement de comprendre la politique migratoire américaine car l'utilisation de l'immigration comme bouc-émissaire peut affecter les relations entre les Etats-Unis et le Mexique, et s'avérer néfaste également pour la politique d'intégration économique continentale présidée par les Etats-Unis. Non seulement la conjoncture actuelle est marquée par une recrudescence de la politique migratoire américaine restrictive, mais il semble également que les positions anti-immigrées se renforcent y compris parmi la minorité hispanique américaine. C'est une attitude aussi bien démocrate que républicaine, ce qui aggrave la situation, et polarise les forces internes, contre tous les hispaniques, en soutenant les comportements racistes. Or tôt ou tard, la politique anti-immigrés affectera aussi les hispaniques qui s'opposent à l'immigration.

La politique migratoire mexicaine quant à elle repose sur une absence totale de restriction, et ne se définit guère comme telle bien qu'elle ait fonctionné comme une soupape face aux pressions économiques, car elle ouvre une porte de sortie à un nombre considérable de pauvres qui se doivent d'émigrer, en raison de l'absence d'opportunités économiques et qui, une fois hors du pays, envoient des sommes importantes en devises ${ }^{6}$. La politique américaine est plus politique, car d'un point de vue formel les frontières sont fermées, mais dès qu'un besoin de main d'oeuvre se fait pressant elles s'ouvrent surtout durant la pleine saison des récoltes.

Migration et définition de la frontière 
Le Mexique et les Etats-Unis considèrent d'un commun accord la frontière comme un espace vide. Cette idée tire son origine des colonisations espagnoles, britanniques et irlandaises. Pour les Espagnols caractérisés par une «mentalité de rentier» (obtenir des terres pour recevoir une rente et un tribut) le nord sauvage n'était pas attrayant. Pour les colonisateurs des premières colonies britanniques, la terre était offerte à l'expansion et n'appartenait à personne. La frontière était ainsi un espace représentant l'aventure.

"Jusqu'à la fin du XIXe siècle les frontières étaient considérées comme des mauvaises terres ou "malpais"- le royaume des serpents à sonnettes, des bandidos et rats du désert " 7 .

Le climat désertique et aride a permis de perpétuer la perception de cette zone comme " un désert sans beauté ni vie " ${ }^{8}$ et un espace vide. C'est ainsi, que très tôt la frontière représenta un écosystème de violence, résultant de l'éloignement des centres du pouvoir, et d'un sentiment de distance par rapport à l'application de la loi. Nombre de frontaliers pensaient que cette zone possédaient ses propres valeurs et sa propre morale.

Cette conception a prévalu dans les deux pays et modelé leurs politiques frontalières. Mais concernant la protection des frontières, il faut rappeler que l'expansion américaine durant le XIXe siècle a privé le Mexique de plus de la moitié de son territoire, et bien qu'il y ait une différence notoire quant aux définitions respectives de chaque Etat à propos de la frontière, le concept d'espace vide joue de part et d'autre.

Pour les Etats-Unis la région frontalière touche au thème de la sécurité nationale alors que pour le Mexique c'est plus une question de souveraineté et de protection patriotique dans le sens et physique et symbolique9. Les Etats-Unis définissent le Mexique comme une arrière-cour et la frontière serait donc une clôture: on ne permettrait sous aucun prétexte que des «étrangers » sautent illégalement cette clôture car ils pénétreraient alors dans une propriété privée. Rappelons que les lois américaines protègent celui qui tue un intrus qui est entré dans son domicile.

Il est probable que ce concept de propriété soit le support philosophique justifiant le fait que les autorités américaines considèrent les émigrants mexicains qui traversent «illégalement » la frontière comme des criminels et par conséquent deviennent sujets à de redoutables sanctions. Ainsi, pour beaucoup de policiers qui protègent la clôture, leur abus d'autorité est une conséquence naturelle puisqu'ils sont protégés même s'ils en arrivent à tuer des immigrants ${ }^{10}$.

Le gouvernement mexicain adopte une posture paranoïaque dans sa perception des Etats-Unis car c'est un colosse qui lui a déjà pris la moitié de son territoire, il ne peut lutter contre lui et il doit le traiter avec précaution.

S'il est évident que cette relation présente des avantages, elle comportent également potentiellement des risques, comme celui d'une invasion culturelle ou bien une domination économique. Cette approche paranoïaque a peut-être motivé une politique ambiguë, passive, située dans une perspective libérale classique de laisser-faire et laisser-passer, où nombre d'événements peuvent se produire dans une zone frontalière militairement non-protégée, où seuls quelques corps policiers agissent. En revanche les Etats-Unis maintiennent des bases militaires situées à une ou quelques heures de « route » des 10 villes frontalières mexicaines les plus importantes.

Tableau I : Populations frontalières/villes frontalières ${ }^{11}$ 
$19701980 \% 1990 \%$

Total U.S. 203302.031226545 .80511248709 .87310

Total Mexique 48225.23866846 .8333981249 .64522

Brownsville, TX 5252284997629896216

Matamoros, Tamps. 1377491887033730329361

McAllen TX 3763666281768402127

Reynosa, Tamps 1373831946574226566336

Laredo, TX 69024914493212289934

Nuevo Laredo, Tamps 148867201690352184138

Eagle Pass, TX 15364214073920651 -4

Piedras Negras, Coah. 4103367444649617843

El Paso, TX 3222674252593251534221

Ciudad Juáres, Chih. 4073705444963478952245

$19701980 \% 1990 \%$

Nogales, AZ 894615683751948924

Nogales, Son. 52108655872610578361

Calexico, CA 1062514412361866329

Mexicali, B.C. 2634983415593043837728

San Diego, CA 697027875538261110.54927

Tijuana, B.C. 2773064295005569875263

L'immigration a contribué à augmenter l'importance de la frontière en créant plusieurs métropoles dans chaque pays. Aujourd'hui, comme on peut l'observer dans le tableau I, 4906.557 de personnes se sont établies dans seize villes frontalières, allant de Calexico avec 18.663 habitants en Californie jusqu'à San Diego, aussi en Californie avec 1110.549 habitants.

Cette croissance accélérée a donné naissance à une zone possédant sa propre autonomie économique, non seulement parce que les expériences passées attirent un grand nombre de personnes mais également parce que sa superficie a créé un marché important. C'est un facteur qui attire l'attention des centres de pouvoir des deux pays, et tient également au fait que la frontière est devenue une zone à risque qui touche un nombre considérable de personnes.

De plus, les conditions d'instabilité économique et politique continentales ainsi que les récessions prolongées des dernières décennies ont provoqué des migrations qui, suite à la « droitisation » de la politique des Etats-Unis, sont perçues comme dangereuses pour la société américaine. Cette dynamique a motivé un changement draconien dans la politique migratoire mexicaine et américaine. Les deux pays ferment leurs frontières au Sud et les maintiennent ouvertes au Nord.

La migration mexicaine aux Etats-Unis

Des trente deux Etats de la République mexicaine, dix-huit "fournissent" des candidats à l'émigration. Dans les années 80 le Guerrero (15,2\%), Michoacan (17,6\%), Hidalgo $(18,0 \%)$, Durango $(18,8 \%)$ et le cas extrême de Zacatecas $(33,8 \%)$ ont connu une perte migratoire supérieure à $15 \%$. 
Une étude faite en janvier 1992 pour le service de l'Immigration et de la Naturalisation américain dans la zone de San Diego, signale que $52 \%$ des Mexicains détenus provenaient des districts fédéraux, Jalisco, Michoacan et Guerrero ${ }^{12}$.

Même si dès les années trente des Etats tels que le Zacatecas et le Durango envoyaient leurs citoyens aux Etats-Unis, on ne peut que constater une augmentation de l'immigration mexicaine depuis les années soixante-dix ainsi d'ailleurs que le taux de pauvreté.

La migration mexicaine résulte d'un processus économique et a servi de soupape économique et même politique étant donné les pressions potentielles qu'un taux de chômage élevé aurait pu provoquer dans le pays ${ }^{13}$. Dès lors on soutient la thèse que les migrants mexicains aux Etats-Unis doivent être considérés non comme des immigrants mais comme des réfugiés économiques et qu'ils ont droit par conséquent à un statut protecteur similaire à celui des réfugiés politiques, car ils sont forcés de partir de chez eux $^{14}$.

La dimension quantitative

En 1942, les Etats-Unis et le Mexique élaborèrent le Programme Bracero qui consistait à « importer » d'une façon temporaire un nombre illimité de travailleurs agricoles. Vers la fin du programme, en 1964, plus de 4,5 millions de mexicains ont travaillé temporairement aux Etats-Unis ${ }^{15}$. Il faut toutefois préciser que ce chiffre peut comprendre des travailleurs ayant été employés plus d'une fois.

Bien que nous ignorons combien de fois en moyenne fut employé chaque individu, il est évident que le programme Bracero n'a pas absorbé toute la main d'oeuvre mexicaine disponible, étant donné le flux migratoire des personnes sans papiers d'identité.

Durant son application il y eu environ 33\% de Mexicains en situation d'être déportés, et pour 1954 seulement, un million d'immigrés sans papiers furent déportés. A cette époque déjà le niveau moyen d'un salarié aux Etats-Unis attirait les Mexicains. Au terme du programme, le nombre de Mexicains déportables augmenta en termes absolus et proportionnels. En 1961 il y avait 29817 Mexicains déportables représentant 33,6\% du chiffre total, en 1976, ils atteignirent 781474 soit $89,2 \%$ du total des personnes déportables ${ }^{16}$.

Le flux de Mexicains avec et sans papiers a créé des conditions de reproduction de ce flux, comme les réseaux de soutien, qui alliés à la mauvaise situation économique qu'a connu le Mexique durant ces douze dernières années a gravement augmenté le chiffre total de Mexicains aux Etats-Unis. Cependant il est difficile de connaître avec exactitude ce chiffre même s'il s'agit de n'obtenir qu'une approximation. Ceci est dû à la définition du recensement qui regroupe les "Latinos" ou hispaniques et à une mauvaise évaluation de ce groupe. D'autre part, il est facile de tomber dans le piège des chiffres exorbitants sans se rendre compte que souvent on utilise des chiffres dont on ignore la source. Lors d'une interview avec le directeur du Département des Services de l'Immigration et Naturalisation (INS) à El Paso Texas ${ }^{17}$, celui-ci avoua que les chiffres qu'on utilise pour calculer le nombre de Mexicains sans papiers aux Etats-Unis ne s'appuie sur aucune base scientifique.

Il apparait de toute évidence que la technique du «guess estimate » a prédominé dans le calcul des Mexicains vivant aux Etats-Unis et des immigrants sans papiers. En 1975, un groupe d'intéressés sans autre référence que leurs propres opinions estimaient à 8 180000 le nombre de Mexicains se trouvant aux Etats-Unis ${ }^{18}$. 
Les chiffres utilisés par la «border patrol » sont gonflés pour exacerber des sentiments anti-mexicains qui lui permettent d'obtenir des augmentations budgétaires. Le plus grave est que de nombreuses recherches sont basées sur ces estimations, ce qui a un effet désastreux, un impact néfaste pour les deux pays et entraîne une politisation intempestive de la question de l'immigration. L'enregistrement d'un chiffre élevé d'immigrés mexicains justifie l'amplification de la militarisation frontalière, et assure également la censure du système économique mexicain.

La «border patrol » insiste actuellement sur le fait que chaque détenu représente un individu bien distinct, et ne prend pas en compte le fait de savoir s'il s'agit ou non d'un récidiviste, gonflant ainsi artificiellement les chiffres. Pour 1992 et 1993 elle enregistrait 1,1 millions de détenus (cf. tableau II) calculant que pour chaque détenu trois immigrants arrivent à pénétrer le pays, ce qui donne un total de 3,3 millions qui s'ajoute aux 1,1 millions, c'est à dire 4,4 millions de Mexicains. Si ce nombre était correcte, plus de $5 \%$ de la population totale du Mexique par an émigrerait vers les Etats-Unis ${ }^{19}$.

L'INS modifie ce chiffre en considérant que ces 4,4 millions rendent compte d'une moyenne de cinq tentatives ce qui réduit le nombre à 880.000 mexicains, chiffres que les autorités migratoires mexicaines semblent accepter.

Une étude récente ${ }^{20}$ accepte les données de détenus provenant de la «border patrol ", qui applique la convention de trois « introduits " pour chaque détenu; mais cette étude établit un chiffre de 2,8 tentatives pour chaque traversée à succès, ce qui donnerait 1 571.428 d'immigrants mexicains sans papiers en 1992.

L'étude de Santibañez fut appliquée seulement à deux postes de Tijuana et peut avoir une influence saisonnière non explicite sur le rapport, le facteur accepté de trois personnes passant la frontière pour chaque détenu, tout comme l'idée utilisée par l'INS de cinq tentatives pour chaque traversée, s'avèrent inconsistants.

Tableau II : Arrestation à la frontière américano-mexicaine

1990*1991 19921993 1994**

Total 206920108185011343321182912647229

Marfa 158710837337644277898371

McAllen 16376861368639011525981376

Del Río 482338746331924373833540

El Paso 3583635836142048179410158070

Yuma 699726798236522366514072

Tucson 970461406735449880289890

San Diego 101475544466544576889930477106

El Centro 650430435293263037117549

* de octobre à décembre

** de janvier à juillet

source : « border patrol »

On peut dire que le nombre d'immigrés sans papiers s'élève entre 880.000 et 1,5 millions bien qu'avancer de tels chiffres demeure toujours un peu risqué. Cependant, l'autre approximation possible consisterait à prendre en compte le nombre de Mexicains qui ont émigré légalement et qui s'élèverait à 4,7 millions en cinq ans ${ }^{21}$. 
Selon ce chiffre, le Mexique " produirait » presque $2 \%$ d'émigrés, ce qui expliquerait le taux de chômage peu élevé rapporté par le gouvernement mexicain (environ 3,5\% en 1993, bien que d'après les leaders ouvriers ce taux atteindrait $20 \%{ }^{22}$ ). Si 1,1 millions de Mexicains intègrent annuellement le marché du travail et que durant un an plus d'un million et demi ont émigré, on aurait donc une perte nette de plus de 400.000 personnes. Si cette tendance persiste, un renversement de la situation sur le marché du travail engendrerait une carence de la force du travail

L'épée de Damoclès qui plane sur les statistiques migratoires réside dans la politisation des chiffres. Ceux-ci peuvent être maniés vers une baisse pour contrecarrer les intérêts conservateurs des Etats-Unis, parmi lesquels certains s'avouent racistes ${ }^{23}$. D'autre part, cette utilisation donnerait une fausse idée sur la véritable ampleur du courant migratoire mexicain vers l'extérieur, masquant la nature des causes qui le provoque et qui peuvent se synthétiser en une mauvaise distribution du revenu et une extrême misère.

Neoliberalisme et concentration de la richesse

D'un point de vue historique, le Mexique a vu sa richesse concentrée entre les mains d'une poignée d'individus. La stratégie poursuivie par le gouvernement : augmenter le profit pour favoriser l'investissement, provoqua une misère extrême et en contrepartie une richesse prodigieuse.

Pour illustrer rapidement les manifestations de cette richesse nous pouvons citer les scandales comme celui provoqué par une réunion de chefs d'entreprises et du Président de la République pendant laquelle chacun d'eux fit un donation de 25 millions de dollars au Parti Révolutionnaire Institutionnel (PRI) au pouvoir depuis 1929 pour créer un fonds partisan ${ }^{24}$.

Tout aussi surprenants sont les calculs des fuites de capitaux ${ }^{25}$ qui au moment le plus crucial de la crise mexicaine de la dette en 1987, s'élevaient à un montant de 107,470 millions de dollars ${ }^{26}$. Il faut savoir que les Mexicains ont soutenu pendant des années le marché des biens-fonds au sud des Etats-Unis. Durant les années soixante dix, les gens appelaient de façon humoristique le centre balnéaire du Texas, South Padre Island, le Nuevo Leon ${ }^{27}$, ou identifiaient deux élégantes tours de San Diego California, comme les Taco Towers de Coronado.

La concentration du capital n'est pas un concept nouveau. Des années durant les économistes attirèrent l'attention sur les effets pernicieux que peut avoir la persistance de cette concentration. En dix neuf ans (de 1950 à 1969) 20\% de la population la plus aisée éleva de $4 \%$ la part du revenu national qu'elle contrôlait pour parvenir à $64 \%$, reléguant dans la misère $70 \%$ de la population la plus démunie.

Le grand projet pétrolier mexicain de la fin des années soixante dix et du début des années quatre-vingt contribua a aggraver le problème de la distribution. Dans les années quatre-vingt-dix, le gouvernement adhéra au neoliberalisme avec l'intention d'aboutir à une alliance avec les républicains des Etats-Unis. L'hypothèse était que cette politique engendrerait des modifications structurelles bien perçues par les architectes de la reaganomanie, et déboucherait sur un immense flux d'investissements vers le Mexique, l'influence des Etats-Unis sur les organismes financiers internationaux, facilitant un traitement plus favorable du Mexique.

Cependant la réalisation de ce projet fut tardive et n'eut pas les effets escomptés car au lieu de résoudre les entraves à l'économie nationale, il rendit le Mexique bien plus 
dépendant des Etats-Unis, et entraîna une concentration du profit, engendrant ainsi une poignée de très riches et une masse de très pauvres; fait dont on devrait avoir honte et qui historiquement peut être considéré comme une tragédie nationale.

La privatisation du secteur public de l'économie dont on parla tant sous l'administration Salinas a "oligarchisé » le Mexique. Trente six familles, membres du Conseil des hommes d'Affaires, possèdent soixante dix groupes économiques contrôlant l'économie mexicaine et font du Mexique leur instrument personnel d'accumulation de richesse. En effet leur profit équivaut à $73 \%$ du revenu des entreprises cotées en bourse $^{28}$. Au début de sa présidence Ernesto Zedillo est confronté au résultat de cette politique ; ainsi il a du dévalué le peso et entamé un programme d'austérité qui devrait entre autre accélérer l'immigration illégale.

<!--SPIP--> Neoliberalisme et concentration de la pauvreté

La contrepartie de la richesse extrême est la misère extrême. Le miracle mexicain qui démarra pendant les années quarante laissa la séquelle d'une misère très marquée. Depuis 1963 déjà, on comptait 30 millions de pauvres dont 24 millions vivaient dans un misère extrême. En 1984, le nombre de personnes vivant dans un état de misère extrême se réduisit, mais se remit à augmenter dès l'année suivante. En 1991, il y avait 51 millions de pauvres et 19 dans une misère extrême. Seulement un an plus tard le nombre de pauvres s'élèvera à 55 millions et à 20 ceux vivant dans des conditions d'extrême misère ${ }^{29}$. Le responsable du développement du programme alimentaire lors de la présidence de Jose Lopez Portillo (1976-1982) déclara en 1980 : «nous ne pensons pas laisser en dehors de la modernisation ces 20 millions de marginaux, non seulement parce que c'est une question de justice, mais aussi parce que le besoin d'élargir le marché se fait ressentir ».

Citant le Programme de formation et Productivité du Secrétariat du Travail et de la Prévision Sociale Balboa Reya ${ }^{30}$ considère qu'en 1991 le Mexique comptait 12 millions de travailleurs se trouvant dans une misère extrême mais si on prend en compte chaque membre de la famille le chiffre s'élève alors à 34 millions de personnes.

Une partie du problème réside dans la définition des facteurs économiques. L'administration de Carlos Salinas de Gortari en adoptant la thèse du facteur travail comme celui qui provoque le plus d'inflation, établit une politique de stabilisation qui freina le revenu et augmenta le nombre de pauvres et les caractéristiques de la pauvreté.

Même s'il n'y a pas de consensus sur les chiffres, différentes études dressent un tableau dramatique. Selon le XIe Recensement Général de la Population et du Logement ${ }^{31}$ de $1990,12,4 \%$ de la population étaient analphabètes $36,4 \%$ des logements n'avaient pas de système d'évacuation des eaux, 20,6\% n'avaient pas l'eau courante et 12,5\% l'électricité.

Concernant la qualité de la vie, il fut révélé, lors de l'Assemblée des représentants de la ville Mexico, que $34 \%$ des enfants mexicains ont une stature et un poids plus bas que la normale et le taux de mortalité infantile est de 12 pour 1000 naissances $^{32}$.

Dans une étude réalisée par une équipe d'économistes du Centre d'Analyse de la Faculté d'Economie de l'Université Nationale Autonome du Mexique on établit qu'en 1977 $57,9 \%$ de la population vivait en situation de pauvreté. Actuellement le chiffre est de 91,1\%, c'est à dire que 78 millions de Mexicains ne peuvent subvenir aux plus simples nécessités". En 198912730.734 personnes c'est à dire 16,2\% d'entre eux vivaient dans 
un extrême pauvreté, en 1992 le chiffre atteignait 24. 496582 c'est à dire 30,1\% de la population $^{33}$.

Une autre approche serait d'envisager la valeur du salaire minimum, qui est obligatoire au Mexique et se révise annuellement en janvier. Selon les économistes de l'UNAM, depuis 1982 année pendant laquelle Salinas de Gortari se chargea de mener la politique économique du régime jusqu'en 1993 le salaire minimum augmenta de 409\%, mais le prix du panier de base intégral (CBI) constitué de 312 biens et services de consommation et d'utilité quotidienne pour une famille de cinq personnes augmenta de 1,388\%. En 1982 le salaire minimum permettait d'obtenir 38\% du CBI, mais en 1993 il ne permettait plus que l'obtention de $13 \%$ seulement du CBI.

Une autre démarche ${ }^{34}$ consisterait a envisager le salaire minimum en termes réels lequel atteignit un maximum en 1976 et commença a décliner pour en arriver en 1985 à se situer au même niveau que celui de 1966 inférieur de 40,1\% à celui de 1976 et de $25,1 \%$ à celui de 1970 . Ce qui signifie que le salaire minimum était revenu au niveau atteint lors de la précédente génération. Mais si l'on considère que le salaire réel continua à diminuer durant les huit années suivantes et qu'en 1993 la débâcle fut complète $^{35}$ on doit en conclure que les mexicains ont perdu là le labeur de plus d'une génération et non d'une décennie comme beaucoup le pensent.

En termes de valeur ajoutée l'apport du travail au PIB diminua également, passant de 37,4\% en 1981 à 26,4\% en 1986 alors qu'aux Etats-Unis il restait constant ${ }^{36}$. Le rapport du Service d'Immigration, précédemment cité, affirma que 79\% des immigrés étudiés, possédaient un emploi avant d'émigrer, bien que ne gagnant en moyenne que $34,36 \$$ par semaine ${ }^{37}$.

La récession prolongée, engendre depuis des décennies des taux de violence et de criminalité sans précèdent. Sans doute est il significatif que les Etats-Unis qui possèdent des taux de violence électorale particulièrement significatifs, soient ceux qui expulsent aussi des populations.

Face à ce sombre panorama, il n'est guère étonnant que les Mexicains aient réfléchi en fonction de leurs aspirations, sur les réussites de leurs compatriotes qui bénéficièrent du Programme Bracero et soient retournés vers le nord ${ }^{38}$.

Migration vers les villes

Le Mexique présente non seulement une dichotomie pauvres et riches, mais aussi entre villes disposant de maigres ressources et campagnes extrêmement pauvres. La misère rurale est la principale cause de la transformation du Mexique en un pays majoritairement urbain, et ce, en quelques décennies. Cette migration interne n'est pas due à la merveille des villes ou bien aux nombreuses opportunités qu'elles offrent, mais à la situation déplorable des campagnes négligées.

La mauvaise répartition du revenu dans les campagnes s'attestait vers la fin des années soixante par le fait que $86 \%$ des propriétés arables n'atteignaient pas cinq hectares et cette partie regroupait $80 \%$ du travail agricole dont la productivité par travailleur était de $213 \$$ à comparer aux $2300 \$$ dans le reste du secteur économique. Sans mentionner le salaire dont beaucoup de travailleurs agricoles se trouvaient dépourvus, d'autres étaient propriétaires (très pauvres) de minuscules parcelles.

La ville était un aimant pour une population sans perspectives de développement en zone rurale et pour laquelle le seul salut possible résidait dans la migration. 
Cependant l'infrastructure urbaine ne présentait aucune capacité d'absorption. Les villes commencèrent à accumuler la misère et se transformèrent en foyers insalubres et en centres confrontés à des problèmes d'environnement avec une infrastructure décrépie.

Les emplois que trouvèrent les migrants dans les villes étaient mal rémunérés comparés à ceux pratiqués aux Etats-Unis. Le salaire minimum est d'environ 37 cents de l'heure alors qu'aux Etats-Unis il s'élève à $4,25 \$$. Aussi il n'est guère surprenant le flux de population ait pris la direction du Nord. La seule différence peut-être est que maintenant plusieurs cycles migratoires se sont développés, comme celui des zones rurales qui évitent les villes et se dirigent directement vers les Etats-Unis.

Le cas des indiens mixtecos de Oaxaca est très instructif. Dans un seul district électoral, plus de 60000 mixtecos sont partis engendrant ce qui semble être deux cycles combinés. L'un d'eux serait constitué par les travailleurs "hirondelles" qui se déplacent vers les Etats agricoles de Sinaloa et Sonora ${ }^{39}$ au Nord Est du Mexique et après avoir ramassé quelques miettes, retournent à leurs parcelles pour les préparer et les laisser à la charge de leurs épouses.

Mais ce cycle s'est apparemment modifié. Un grand nombre de mixtecos a immigré vers la basse Californie ou se sont créées d'importantes colonies. Ces dernières émigrent souvent vers le Nord, créant un autre cycle qui consiste en un retour vers les colonies de basse Californie, notamment la ville de Tijuana. Beaucoup de gens s'installent alors aux Etats-Unis et ne reviennent pas. L'importance de cette migration est telle que le candidat du PRI voyage jusqu'en basse Californie lors de sa campagne pour l'élection au poste de Gouverneur. D'autres en revanche restent maintenant à Oaxaca.

Les villes moyennes mexicaines, y compris les villes frontalières ne peuvent plus retenir les migrants. Ceci s'explique autant par le manque de travail que par l'absence de logement et services urbains. Paradoxalement, le départ des Mexicains convient au gouvernement afin d'alléger les pressions sur les villes démunies ${ }^{40}$.

La récente migration vers les Etats-Unis

L'incapacité de l'économie mexicaine à créer des emplois, ajoutée aux effets de la migration passée ont provoqué une augmentation substantielle de l'émigration vers les Etats-Unis.

Vernez et Ronfeldt ${ }^{41}$ ont établi que depuis 1976 l'immigration légale mexicaine s'était stabilisée à une moyenne de 66000 personnes par an. Cependant il semble que ce chiffre ait considérablement augmenté en partie à cause des changements légaux intervenus aux Etats-Unis qui redistribuèrent les quotas de l'immigration et facilitèrent la régularisation des personnes qui vivaient déjà dans le pays. Entre 1986-1987, 3,1 millions de Mexicains régularisèrent leur situation migratoire à la suite de la loi Simpson-Rodino. Les nouveaux quotas entraînèrent 1625.235 immigrants légaux entre 1990-199142. Si l'on ajoute la migration légale au scénario, dans lequel nous avons 880 000 migrants sans papiers d'identité par an, nous arrivons à un total de 3385.235 migrants qui représentent plus de $4 \%$ de la population mexicaine.

Pour Corona $\mathrm{a}^{43}$ le "phénomène migratoire est devenu plus hétérogène, complexe, et plus ample " incluant même des zones du Mexique qui n'avaient auparavant jamais "produit » de migrants. Ce phénomène s'est accéléré durant la décennie 1980-1990, s'élevant à un total de 14,094 millions de Mexicains, dont 13,6\% représentent les 
habitants d'origine et leurs descendants issus du territoire qui passèrent du Mexique aux Etats-Unis au XIXe siècle.

Corona démontre qu'un pourcentage élevé retourne au Mexique après avoir habité aux Etats-Unis au moins huit ans. Cependant, il semble que la migration mexicaine soit en train de changer. Au lieu de l'image du mexicain pauvre et illettré, le migrant a de plus en plus un niveau éducation élevé. Ce sont des familles entières qui émigrent et au lieu d'aller et venir elles semblent disposer à s'installer aux Etats-Unis ${ }^{44} \mathrm{Si}$ cette affirmation s'avère exacte et si l'on tient compte des 3,3 millions de mexicains qui émigrent tous les ans, il devait y avoir en 1995 27,2 millions de Mexicains aux Etats-Unis.

La migration produit également des complications sociales, par exemple le démembrement de la cellule familiale provoquée par la migration de l'un des membres de la famille, normalement le chef de famille, incitant les autres membres à émigrer et à se rassembler. Ainsi on estime que $30 \%$ des migrants aux Etats-Unis sont des enfants et que $12 \%$ ont moins de cinq ans. Bon nombre de ces enfants sont sans domicile fixe et émigrent afin de retrouver leurs parents. Selon l'Organisation Internationale du Travail il y a 11000 enfants qui travaillent aux Etats-Unis au mépris des lois ${ }^{45}$.

En général, les migrants mexicains se rapatrient et évitent la naturalisation peut-être parce qu'ils pensent retourner un jour au Mexique. S'ils changeaient d'attitude leur poids politique pourrait augmenter d'une façon considérable.

Aujourd'hui les exemples d'influence sur la politique locale commencent à abonder comme le démontre le cas du Texas. Le cas de Henry Cisneros est à ce propos très révélateur. Il fut Maire de San Antonio, se proposa durant la campagne électorale de Bill Clinton d'être le lien avec les Hispaniques, il est aujourd'hui Secrétaire du Logement et du Développement Urbain.

Les millions de Mexicains ou de personnes d'origine mexicaine vivant aux Etats-Unis se concentrent dans quelques Etats qui, par pure coïncidence, sont ceux qui pèsent le plus électoralement. Les Mexicains enregistrent un taux de croissance, plus élevé que celui de la moyenne nationale si l'on ajoute la croissance naturelle à celle due à l'immigration; de sorte que d'ici une décennie le poids politique de la communauté mexicaine sera élevé et pourra devenir un élément crucial pour la résolution de certaines questions électorales.

Lire la suite

\section{NOTES}

1. Professeur à l'Université Texas d'El Paso (UTEP).

2. Dans le cas des migrants d'Amérique Centrale la situation est encore plus dramatique. On dénonce le viol systématique de femmes d'Amérique centrale qui traversent la frontière. On peut en voir un exemple dans le film « el Norte ». Ce phénomène diffère toutefois du cas mexicain et n'est pas le sujet de cet article

3. Le Congrès des Etats-Unis prévoit dans sa loi sur le crime une augmentation de 6000 agents de la «border patrol » durant les cinq prochaines années qui viendront s'ajouter 
aux 4000 agents déjà existants. Associated Press, « Border Patrol Stretched Too Thin », El Paso Times July 17, 1994, 7B.

4. Zuniga Victor, « Los Locos del Barrio o la Ostentacion del Estigma : Identidad Social y Frontera en una Pandilla de Matamoros, Tamaulipas », Rio Bravo., Vol.1, n¹., 1991, p. 47-68.

5. Calavita Kitty, «California's « Employer Sanctions »: The Case of the Disappearing Law, San Diego, University of California, San Diego, Center for U.S.-Mexican Studies. Research Report Series, 39, 1982.

6. On observe une augmentation des envois de devises, qui sont passés de 1262 dollars en 1980 à 2300 dollars en 1985 et à 3151 dollars en 1990.

7. Barry Tom \& Sims Beth., The Challenge of Cross Border Environmentalism. The U.S.Mexico Case, Albuquerque, Resource Center Press, 1993, p. 19. La description de Sonnichsen à propos du déménagement du légendaire juge texan Bean vers Mesilla ,Nouveau Mexique dit :" the Bean Brothers always heades for a frontier community on the make where pushing Americans could get theirs without too much regulation and interference".

8. Barry Tom \& Sims Beth, op. cit., p. 19.

9. Il existe encore au Mexique des peurs d'une invasion idéologique et culturelle rappelant la décenniedes années 70, quand le gouvernement tenta d'interdire les affiches publicitaires en anglais.

10. Les policiers sont apparemment protégés par un code d'honneur même s'ils abusent des citoyens. Bien sur ceci s'applique également à l'abus des migrants.

11. Source : David E. Lorey, United States-Mexico Border Statistics Since 1900. 1990 Update, (Los Angeles :UCLA,1993), table S100.

12. U.S. Immigration and Naturalizaton Service, Recent Upsurge in Apprehensions of Mexican Nationals for Illegal Border Crossings in the San Diego Sector : A Look at Causative Factors. Mexico City Office. American Embassy, sans date, p. 4.

13. Cothran, Dan A, Political Stability and Democracy in Mexico, Westport, Praeger, 1994, p. 4.

14. Schmidt Samuel, « Migracion o refugio economico. El caso mexicano », Nueva Sociedad, n 127, sept.-oct. 1993, pp. 136-147.

15. Vernez Georges, Ronfeldt David, « The Current Situation in Mexican Immigration ", Science, Vol. 251, march 8, 1991, p. 1189.

16. Diez Canedo, Juan., La Migracion Indocumentada de Mexico a los Estados Unidos. Un Nuevo Enfoque, Mexico D. F, Fondo de Cultura Economica, 1984, p. 30, tableau 1. Cet auteur considère également la migration comme un problème économique.

17. Entretien avec Alfred Giugni, 30 mars 1993.

18. Diez Canedo, Juan., op. cit., p. 31.

19. La « border patrol » aurait modifié dernièrement son critère de 3 pour 1 pour celui de1 pour 1 , afin de ne pas montrer que la première comparaison leur donnait une image d'inefficacité.

20. Je remercie les auteurs pour m'avoir procuré une copie préliminaire de la conférence. Santibañez, Jorge, Valenzuela, Javier y Velasco, Laura, Migrantes Devueltos por la Patrulla Fronteriza, presentado en la Conferencia Facetas de la Violencia Fronteriza, Universidad de Texas en El Paso, Mayo 3-4, mimeo, 1993.

21. U.S. Department of Justice, Immigration an Naturalization Service, INS Fact Book, U.S. Government Printing Office, 1993. 
22. De sérieuses réserves existent quant à la fiabilité statistiques officielles mexicaines ainsi que sur le taux de chômage Il semble que la méthode suivie pour mesurer ce dernier ait tendance à le sous-estimer.

23. A San Diego, un groupe a surgi vers la fin des années 80 . Il allait à la frontière pour « chasser » les Mexicains. Un juge californien déclara récemment innocents deux individus qui avaient molesté des Mexicains. C'est aussi en Californie que l'on a approuvé la proposition 187 qui n'autorise pas les immigrants sans papiers a bénéficier des prestations du système éducatif et des services médicaux convertissant les prestateurs de ces services en informateurs du Service de la migration. L'application de la loi fut suspendue par la Cour suprême, mais le thème de l'immigration a investi le débat politique qui tend vers une fermeture de la frontière et la suppression des services dont peuvent bénéficier les immigrants avec ou sans papiers.

24. Rodriguez Castañeda Rafael, « Borrego, 29 magnates y el Presidente de las Republica », Proceso, n 853, Marzo 8, 1993, p. 13. John M. McClintock du Baltimore Sun indique que les millions de dollars que l'on essaya de réunir, était de loin supérieure aux 300 millions de dollars que coutèrent les campagnes de Clinton, Bush et Perot réunies. L'auteur conclut :"dans un pays où la moitié de la force de travail gagne 9,40 dollars par jour le fait que 30 hommes réunissant \$750 millions en une soirée est la confirmation cynique de ce que n'importe quel mexicain pense d'un gouvernement denommé « la mafia ».

25. Il n'existe aucune source qui établisse le montant des capitaux qui quitte le Mexique et d'autres pays d'Amérique Latine. En 1976, la dette total s'élevait à 19600 millions, 27.296 millions sortirent du pays. (Samuel Shmidt, The Deterioration of the Mexican Presidency, Tucson, University of Arizona Press, 1991, p. 119). De 1976 à 1984 27milliards ont quitté le pays (Frieden, Jeffrey A., « Winners and Losers in the Latin American Debt Crisis » in Barbara Stallings, Robert Kaufman (eds.) Debt and Democracy in Latin America, Boulder, Westwiew, 1989, p. 31.).

26. Gurria, Jose Angel, « Debt Reduction and the "Virtuous Circle" », El Financiero International, July 22, 1991, p.15.

27. Dans ses mémoires un ex gouverneur de Nuevo Leon Luis Farias déclare : « on prétend que pour être considéré originaire de Monterrey il faut avoir une maison dans l'Isla del Padre, car pour s'inscrire comme candidat pour Monterrey on nous demande une maison la-bas » (Farias Luis M., Asi Lo Recuerdo. Testimonio Politico, Mexico, D. F., Fondo de Cultura Economica, 1992, p. 170.).

28. Acosta, Sarah, « Mexico Inc., the Corporate Elite », Hispanic Business, Agosto 1992, p.18-30.

29. Balboa Reyna, Flor de Maria, « Antes de que la verdad Irrumpa », La Jornada Laboral, Agosto 30, 1991, p.4-5.

30. Op. cit., p. 5

31. Les données préliminaires au recensement furent remises en question car le dénombrement de la population de la presque totalité des villes était très bas. L'erreur provenait apparemment du fait l'on ne comptabilisa pas les résidents irréguliers qui dans nombre de cas étaient aussi nombreux que les habitants de la ville. Dans la ville frontalière Ciudad Juarez, Chihuahua, on compte 85 colonies irrégulières et selon les autorités municipales presque plus de 500000 personnes des 750000 inscrites lors du recensement.

32. Reyes Morales, Rico, « Pride Mayor Presupuesto la ARDF Para Gasto Social y de Salud », El Financiero, Abril 8, 1993, p. 19. 
33. Corro Salvador, «Vertiginoso Crecimiento de la Pobreza en el Sexenio », Proceso, $\mathrm{n}^{\circ}$ 854, Marzo 15, 1993, p. 30-31.

34. Martinez, Ifigenia, Algunos Efectos de la Crises de la Distribucion del Ingreso en Mexico, Mexico D.F., UNAM, 1989, p.46.

35. Banamex, Review of the Economic Situation of Mexico, Mexico D.F., august, tableau 7, 1993, p.330.

36. Martinez, Ifigenia, op. cit., p. 37-38.

37. Department of Justice, Immigration an Naturalization Service, INS Fact Book, U.S. Government Printing Office, 1993, p. 4.

38. Herrera Sobek Maria, The Bracero Experience. Elitelore versus Folklore, Los Angeles, University of California Press, UCLA Latin American Studies Center Publications, 1979.

39. Sonora est à la frontière avec les Etats-Unis.

40. World Bank, Urban Policy and Economic Development. An Agenda for the 1990s, Washington D.C., 1991.

41. Vernez Georges, Ronfeldt David, op. cit.

42. U.S. Department of Justice, Immigration an Naturalization Service, INS Fact Book, U.S. Government Printing Office, 1993.

43. Corona Vasquez Rodolfo, »Una estimacion del crecimiento de la poblacion de origen mexicano que reside en los Estados Unidos, 1850-1990 », Rio Bravo, Vol.III, n² 2, spring, p. 92.

44. Vernez Georges, Ronfeldt David, op. cit.

45. Données présentées et remises en question par Jesus Rodriguez, Directeur des Organismes Internationaux de la Commission National des Droits de l'homme au Foro de Consulta Frontera Norte de la Comission des Affaires frontalieres de la Chambre des Deputés, Monterrey, Nuevo Leon, 13-14 avril, 1993.

INDEX

Index géographique : Etats-Unis, Mexique

Mots-clés : centre de rétention, expulsions, frontières 